\title{
Image identification based on ARMA model
}

\author{
Ya-Qiong Yan $^{\dagger}{ }^{1,3}$, Cai-Cheng Shi ${ }^{2}$ and Zhi-Yi He ${ }^{3}$ \\ ${ }^{I}$ The Second Academy of China Aerospace, Beijing 100039, China \\ ${ }^{2}$ Beijing Institute of Technology \\ ${ }^{3}$ Beijing Institute of Remote Sensing Device \\ E-mail:girlyanyaqiong@163.com
}

\begin{abstract}
An image identification method based on ARMA model is introduced in this paper Firstly, build the ARMA model for the most common image of a certain kind and then make predictions for the image to be distinguished line by line, and calculate the high order statistics of the residuals at the same time. The simulation results show that according to the high order statistical properties of the residual, different kinds of images could be distinguished and the specific area represented by the model could be located in the image.
\end{abstract}

Keywords: Image Identification; ARMA model; Residuals.

\section{Introduction}

System Identification means to model the system by known observation information of the system. It purposes to estimate the unknown parameters of the system under some orientation [1]. It is widely used in Modern Control theory, Signal Processing, Biomedical Science and Society Economics to analysis their cause and effect relationship, pattern of motion and so on. It is a commonly used method for building mathematical model for the system.

System identification theory is divided into two categories: Parametric model identification method and Nonparametric model identification method. Nonparametric model identification method is a classic identification method which outputs a Nonparametric model. Assuming that it is a linear system, it can be modelled by frequency response method, pulse response method, step response method to analysis without prior conformation on its specific structure.

Parametric model identification method also called modern identification method must assume a structure for the model. The parameters of the model are determined by minimizing the error criterion function between the model and the process. Typical Parametric model identification methods are the least squares method, Random approximation (Steepest descent method), Maximum likelihood method and so on.

Image identification is the cornerstone of the computer vision research in 
solving the complex visual problems such as tracking, segmenting, scene understanding and so on. The first step to analysis a complex scene is to determine what objects are in the image (classification), or which position are they located. Therefore accurate and effective image identification means a lot in computer vision field.

Traditional image identification is based on the pixels with obvious characteristics, such as Harris operator, FAST (Features from Accelerated Segment Test) operator, LOG (Laplacian of Gaussian) operator, HOG (Gradient Histogram ) and so on. In this paper, the image is equivalent to a discrete time series. The Auto Regressive Moving Average (ARMA) model is used to build models for the specific scenes with parameter determination process. Meanwhile it is compared with HOG algorithm in identifying different scenes in one image and detecting the location of the specific scene. Simulation results shows that ARMA model could distinguish different scenes and locate at the same time with better effect than HOG algorithm.

\section{ARMA model}

ARMA model is a commonly used prediction method on time series proposed by Box and Jenkins [2]. The basic idea is to research on the model of a discrete-timeseries which varies with time and correlates with each other to obtain its inner structure essentially. Nora Muleret al. [3] proposed a approach to avoid the propagation of the effect of one outlier when computing the innovation residuals of the ARMA model. Ameiet al. [4] constructed a discrete-time series based on the empirical recurrent rates (ERRs) of the assumed Poisson process, computed sequentially at equidistant time intervals during the observation period. Stef Van Buuren [5] presented a method that can be extended in fitting an Auto Regressive Moving Average (ARMA) model to a latent component instead of to the observed data.

\subsection{Model definition}

ARMA model by Box and Jenkins is defined as the deterministic relation:

$$
Y_{t}=\zeta+\sum_{j=0}^{q} \beta_{j} E_{t-j}+\sum_{i=1}^{p} \alpha_{i} Y_{t-i}
$$

Where $\zeta$ is the intercept and defined equal to $0, \sum_{i=1}^{p} \alpha_{i} Y_{t-i}$ is the autoregressive (AR) part, $\sum_{j=0}^{q} \beta_{j} E_{t-j}$ is the moving average (MA) part with $\beta_{0}$ fixed as 1 , and $E_{t} \sim \aleph(0, \gamma)$ is "white noise" with $E_{t}$ being mutually independent for all $t$. The parameters $\mathrm{p}$ and $\mathrm{q}$ are real-value model order for the AR part and the MA part, respectively. 


\subsection{Model construction}

\section{1) Data preprocessing}

The image data is colored noise, and thus does not follow the Gaussian distribution. Normally, the data is preprocessed with methods such as partial differential, mean elimination, normalization in horizontal, to achieve approximate Gaussian distribution for signal. The formula is defined as follows:

$$
x_{t}=z_{t+N}-z_{t}
$$

Where $z_{t}$ is the original image, $\mathrm{N}$ the pixel interval, and $x_{t}$ the value of partial differential.

\section{2) Model Parameter determination}

The commonly used AIC principle is adopted as the order determination method. Basically, AIC applies the classic maximum likelihood estimation (MLE) as its estimation principle. This technique stands for an information theoretic criterion and is an estimate of a measure of fit of the model [6]. It is used in determining the order of an AR, MA, and ARMA model, which is a measure of the probability of the estimated model fitting the observed data; and the number of independently adjusted parameters within the model. For a time series of ARMA (p, q) model, we could make a general judgment on its order by observing the ACF and PACF plots. Then, by modeling on different pairs of (p, q) from low order to high order to estimate parameters and orders, and comparing the AIC values of different models, the most proper model can be obtained under the chosen criteria.

The parameter is determined by a single background image such as sky, sea or ground and so on.

\section{3) Model examination}

For ARMA (p, q) model is built on the assumption that the random disturbance is a Gaussian white noise, the model can be examined by the determining whether the residual is white noise. If it is in line with the white noise's characteristics, the model constructed is proper, otherwise it is to be revised [7].

The examination on residual can be done by Autocorrelation function and Histogram distribution.

\section{Simulation}

This section is divided into three parts, Part1 shows the ARMA model 
construction and examination for two categorizes of images. Part 2 shows how the known ARMA model identifies different scenes in a image and locates it. Part 3 shows the effect of HOG algorithm in identifying different scenes in a image.

\subsection{ARMA model construction}

Take the most common sky and grass image for example as shown in Fig.1 and Fig.2, partial differential calculated according to the formula (2), the interval is 2 . The ARMA models for them are shown in formula (3) and (4) respectively.

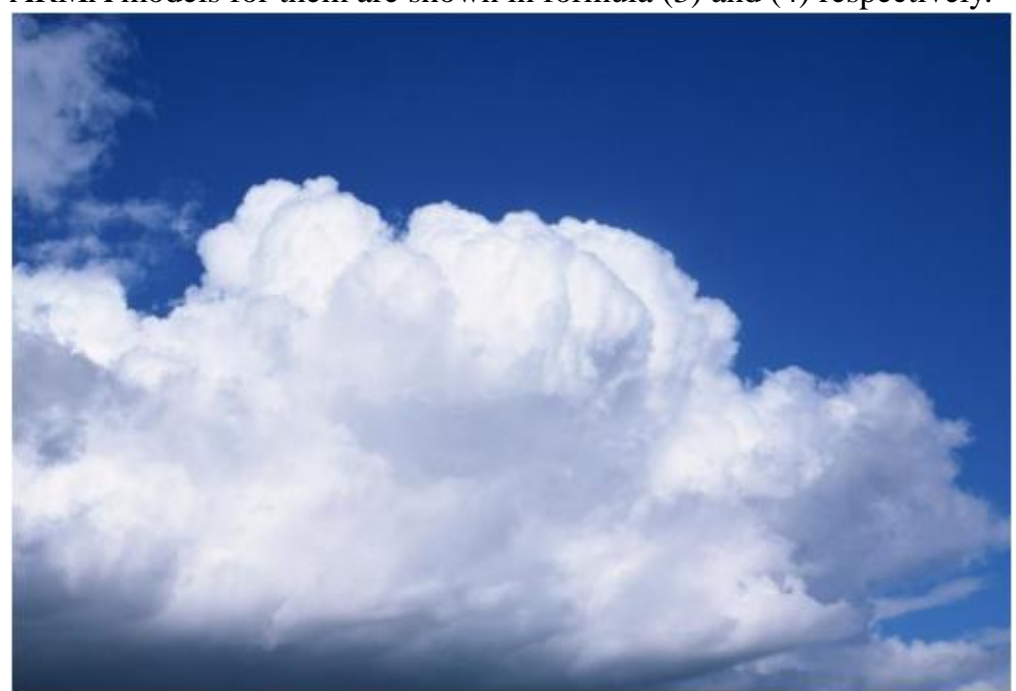

Fig.1 Sky and cloud image

Fig2.Grass image

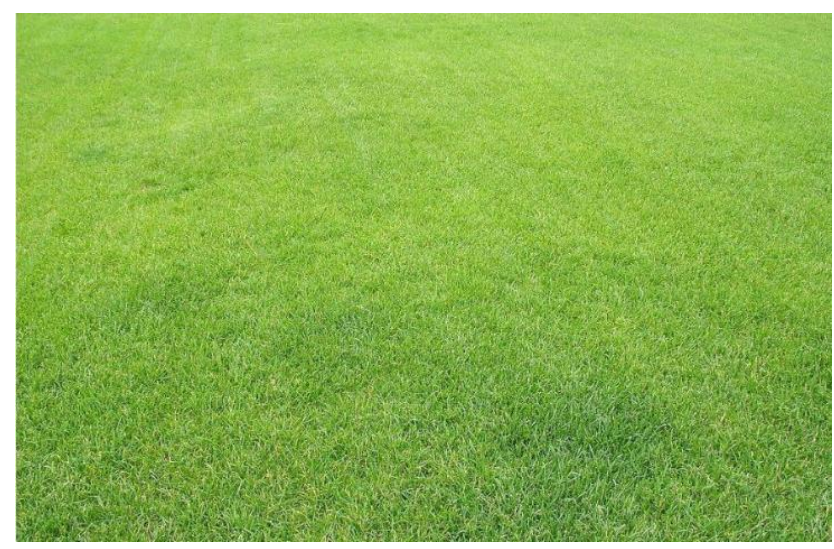

Discrete-time ARMA model for Fig.1: $\quad A(z) y(t)=C(z) e(t)$

$\mathrm{A}(\mathrm{z})=1-2.498 \mathrm{z}^{-1}+3.288 \mathrm{z}^{-2}-3.343 \mathrm{z}^{-3}+2.265 \mathrm{z}^{-4}-1.101 \mathrm{z}^{-5}+0.497 \mathrm{z}^{-6}-$ 
$0.1075 \mathrm{z}^{-7}$

$\mathrm{C}(\mathrm{z})=1-1.028 \mathrm{z}^{-1}+0.3922 \mathrm{z}^{-2}-0.09819 \mathrm{z}^{-3}-0.7648 \mathrm{z}^{-4}+0.4485 \mathrm{z}^{-5}$ $-0.1514 \mathrm{z}^{-6}+0.212 \mathrm{z}^{-7}$

The residual's Autocorrelation function and Histogram distribution for Fig.1 is shown in Fig.3 from which we can see the residual distribution follows Gaussian distribution. Then it can be concluded that this model is properly constructed.
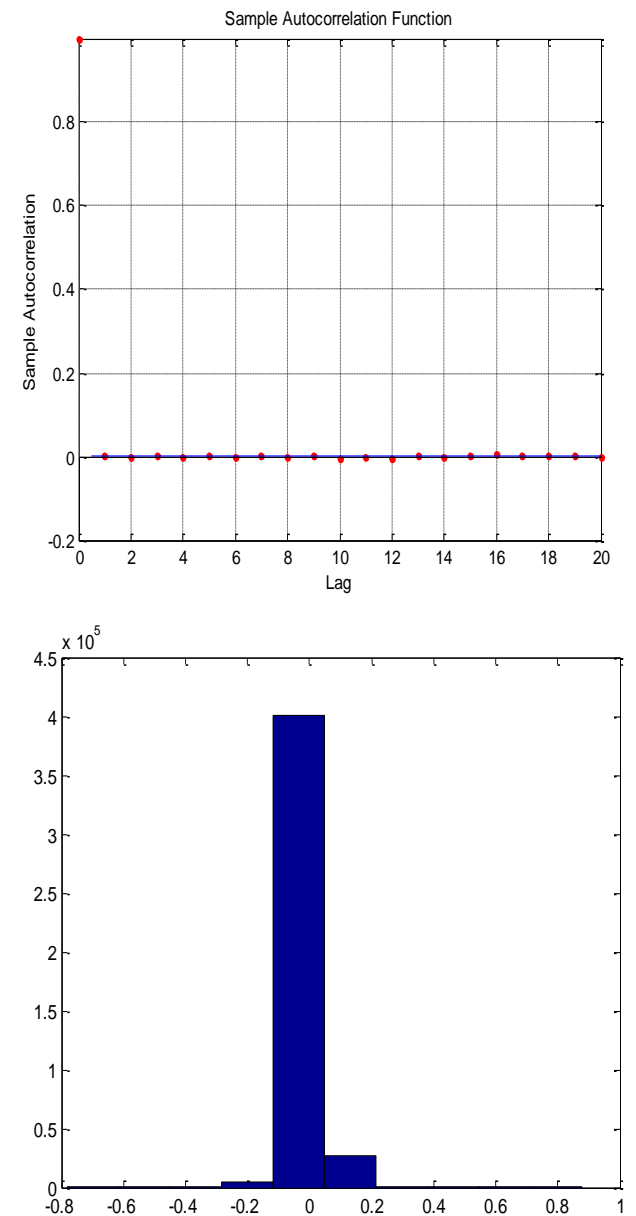

Fig3. Residual's autocorrelation function and histogram distribution

Meanwhile the ARMA model for Fig. 2 can be obtained:

Discrete-time ARMA model for Fig.2: $\quad A(z) y(t)=C(z) e(t)$ 
$\mathrm{A}(\mathrm{z})=1-1.264 \mathrm{z}-1+0.696 \mathrm{z}-2-1.199 \mathrm{z}-3+0.3991 \mathrm{z}-4+0.3287 \mathrm{z}-5+$ $0.04537 \mathrm{z}-6-0.006737 \mathrm{z}-7$

$\mathrm{C}(\mathrm{z})=1-1.094 \mathrm{z}-1-0.3557 \mathrm{z}-2-0.0657 \mathrm{z}-3-0.2655 \mathrm{z}-4+1.276 \mathrm{z}-5$

- $0.1015 \mathrm{z}-6-0.3929 \mathrm{z}-7$
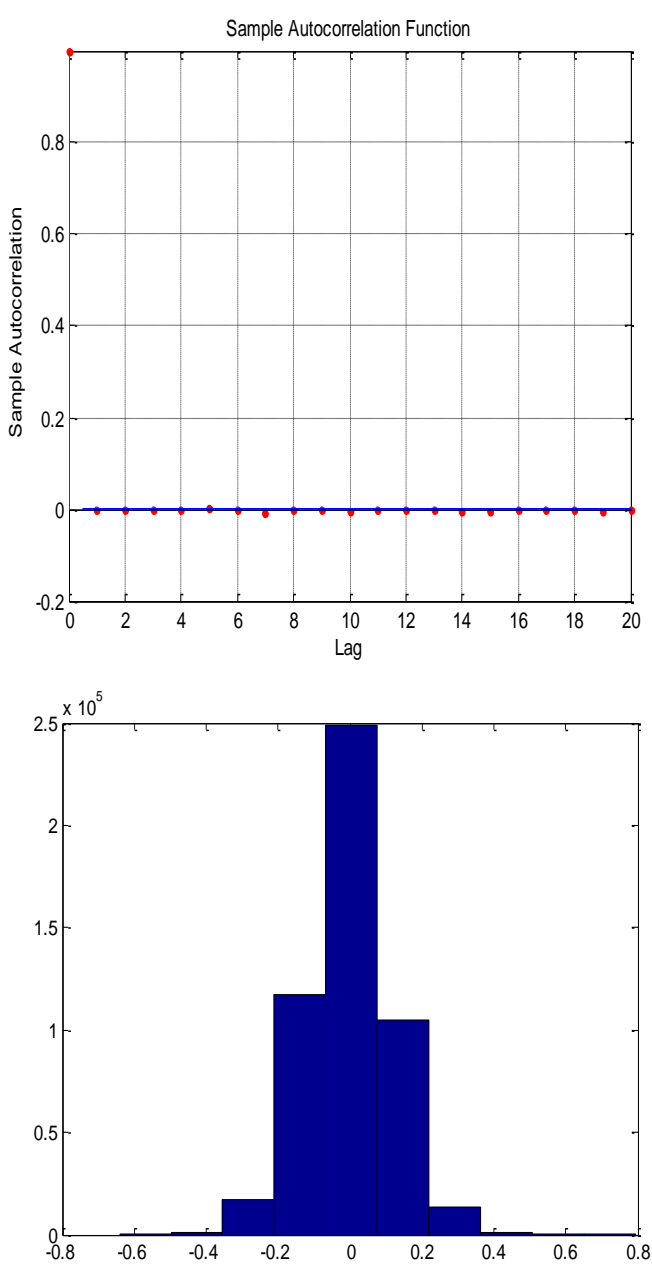

Fig4. Residual's autocorrelation function and histogram distribution

\subsection{ARMA model identification}

Take the common mixed image of the sky and grass shown in Fig.5. 


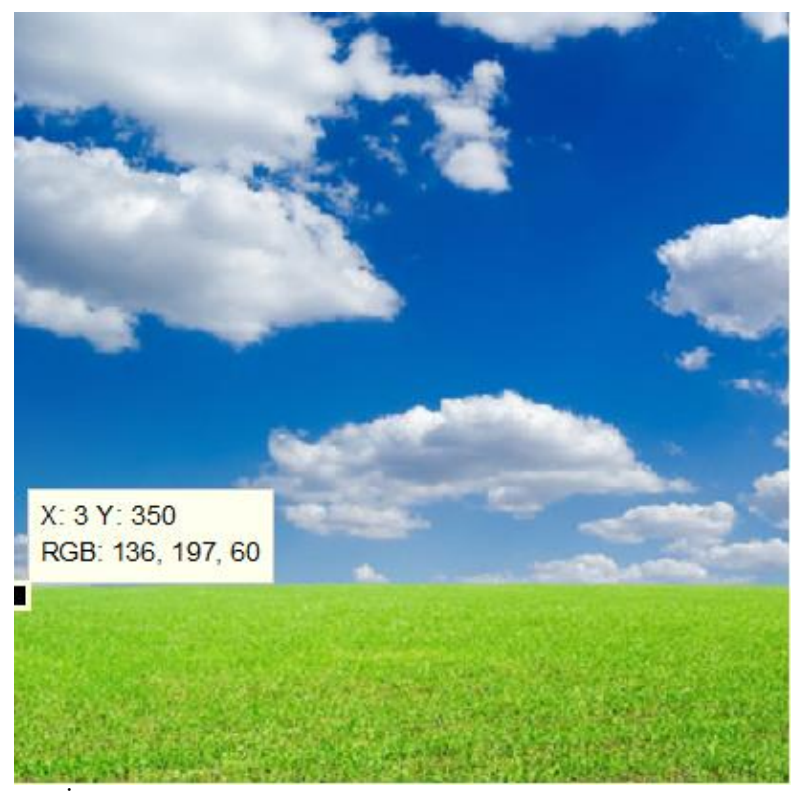

Fig.5.Sky and grass image

Predict the image in Fig.5 with the ARMA model of Fig1 shown in formula (3), and then calculate the MSE (mean square error) of the prediction residual in one row. The MSE of the prediction residual are shown in Fig.8, respectively, in which the horizontal axis represents the row number of Fig.5 and the vertical axis represents the residual's MSE of each row.

As is shown in Fig.6, from the 350th row of image in Fig.5, the residual's MSE changes sharply, so 1th-349th row is sky and cloud background, and the remaining belongs to other background. The actual boundary position of image is 350th as shown in Fig.5. The simulation results show that the MSE of the prediction residual obtained from the sky's ARMA model could distinguish the sky from other parts in one image accurately. 


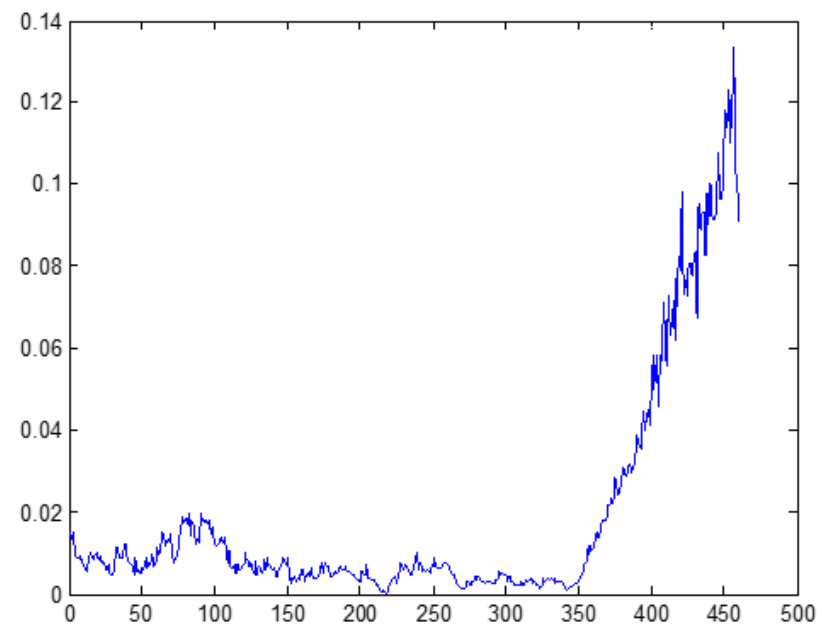

Fig6.Residual's MSE by the sky and cloud model

The same prediction process could be done with the grass's ARMA model of Fig. 2 shown in formula (4). The different scenes of the image could be distinguished by the prediction residual's MSE in Fig.7, which shows the same divided line located at about 350th row as shown in Fig.6.

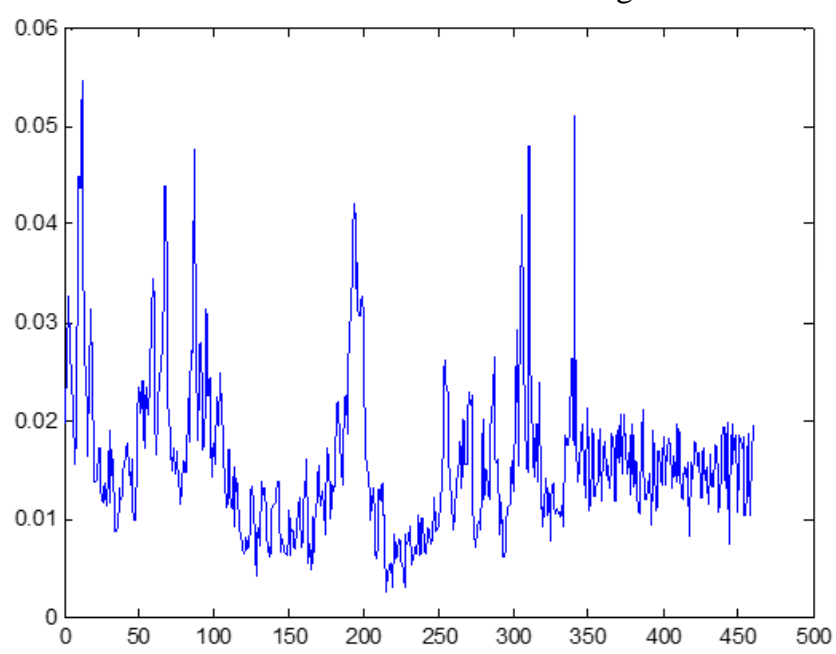

Fig7. Residual's MSE by the grass model

For the difference between the ARMA model of Fig1 and Fig.2, predict the grass part in Fig.5 with the Sky and Cloud (Fig.1) model will result a residual dissatisfied with the Gaussian distribution. Therefore the high order moments of the residual which is a measure of Gaussianity could be regarded as an index to distinguish the sky part and the grass part. Same results could be concluded 
when applying the specific model to more images.

\subsection{HOG algorithm identification}

Fig. 8 is the image identification result by HOG algorithm. Extract the HOG characteristic of Fig.1 and Fig.2, find the difference between them and the HOG characteristic of Fig.5 by row, and then calculate the variance of the difference. In Fig. 8 horizontal axis represents the row number of Fig.5, the vertical axis represents the difference variance of each row, the dotted line is the difference variance between HOG characteristic of Fig.2 and Fig.5 and the solid line represents the difference variance between HOG characteristic of Fig.1 and Fig.5. It can be seen from Fig. 8 that the first 160 rows are the sky and cloud part and the last 150 rows are the grass part which is consistent with the distribution situation in Fig.5. However the middle 100 rows cannot be distinguished from Fig. 8 which is not so satisfying compared with the distinguish result of ARMA model.

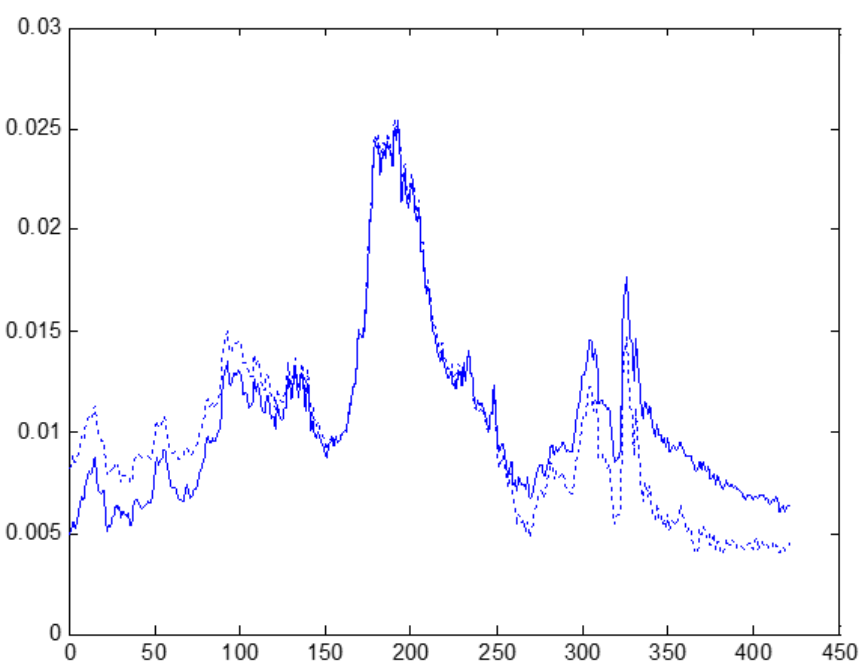

Fig.8 Residual's MSE by HOG algorithm

\section{Conclusion}

ARMA model is proved to be useful and accurate in identifying different kinds of images. Besides, relatively little priori-information is required to implement image identification, which makes it robust and applicable for a wide range of application. Further research will be focused on the image with additive noise, system order lower and so on. 


\section{Acknowledgement}

Work partially supported by grant $2-4570.5$ of the Swiss National Science Foundation.

\section{Reference}

1. Boutalis Y, Theodoridis D, Kottas T, et al. System Identification and Adaptive Control [J]. Advances in Industrial Control, 2014.

2. Box G E P, Jenkins G M, Reinsel G C. "Time series analysis: forecasting and control". John Wiley \& Sons, 2011.

3. By Nora Muler, Daniel Pẽna and V'ictor J. Yohai. "ROBUST ESTIMATION FOR ARMA MODELS”. The Annals of Statistics2009, Vol. 37, No. 2, pp.816-840

4. Amei Amei, Wandong Fu, Chih-Hsiang Ho. "Time Series Analysis for Predicting the Occurrences of Large Scale Earthquakes". International Journal of Applied Science and Technology. Vol. 2 No. 7, 2012 pp.64-75

5. Stef Van Buuren. "Fitting ARMA Time Series by Structural Equation Models". Psychometrika Vol.62 pp. 215-236

6. Akaike H. "A new look at the statistical model identification". Automatic Control” IEEE Transactions on, 1974, 19(6): pp.716-723.

7. Wang Zhenlong, Hu Yonghong. Application of Time Series Analysis [M]. Beijing: Science Press. 2007. 